\title{
Valoración de la influencia de un programa de acompañamiento directivo sobre la percepción y satisfacción laboral de docentes y directivos*
}

\section{Assessment the influence of a school administration accompanying programme to teachers as well as the impact both on the teachers and the school administrators expressed on their perception and job satisfaction levels}

\author{
IngRid Yannine Bravo Carrasco \\ Universidad de Concepción, Chile \\ ORCID: http://orcid.org/0000-0002-5697-2605 \\ Alejandro Díaz Mujica \\ Universidad de Concepción, Chile \\ Claudio Enrigue Navarrete Bustos \\ Universidad de Concepción, Chile \\ María Victoria Pérez Villalobos \\ Universidad de Concepción, Chile \\ Carol Andrea Cuevas Tamarín \\ Universidad de Concepción, Chile \\ Carolina Nova Olave \\ Universidad de Concepción, Chile \\ Marcelo Andrés Albornoz Medel \\ Universidad de Concepción, Chile
}

\footnotetext{
a Autor de correspondencia. Correo electrónico: ingridspring@gmail.com

Para citar este artículo: Bravo, I., Díaz, A., Navarrete, C., Pérez, M., Cuevas, C., Nova, C., \& Albornoz, M. (2017). Valoración de la influencia de un programa de acompañamiento directivo sobre la percepción y satisfacción laboral de docentes y directivos. Universitas Psychologica, 16(2), 1-10.

https://doi.org/10.11144/Javeriana.upsy16-2.vipa
}

\section{RESUMEN}

El objetivo de esta investigación es valorar el "Programa de desarrollo de habilidades de acompañamiento directivo" (PAD), analizando su influencia sobre (1) la percepción y satisfacción de los docentes y (2) la percepción de los directivos sobre su labor de acompañamiento y la satisfacción percibida en los docentes. Se aplicó un diseño cuasiexperimental. Participaron 12 directivos y 16 docentes de cuatro centros educativos de la Región del Bío-Bío, Chile. Se concluye que el PAD tiene un efecto de mejora en (1) la percepción y satisfacción de los docentes, y (2) en la percepción y satisfacción percibida por los directivos en los docentes, por los procesos de acompañamiento realizados. Las dimensiones del PAD que obtienen mayores puntuaciones son apoyo (en los docentes) y sistematicidad (en los directivos).

Palabras clave

acompañamiento; directivo; satisfacción; habilidades

\section{ABSTRACT}

The main objective of this research is to validate "The board of directors accompaniment skills programme to teachers" (BAP), analysing its influence over (1) the perception and satisfaction among the teachers and (2) the perception the board of directors showed about their 
accompaniment work and the perceived satisfaction among the teachers. A quasi-experimental design was applied where twelve participants of four boards of directors coming from four schools of the 8th Region plus sixteen teachers participated. It is concluded that the BAP has an improvement effect over both the teachers' and the board of directors' perception and the satisfaction among the board of directors about the accompaniment process they perform. The dimensions that obtain major punctuations are a support (in the teachers) and systematicity (in the executives). The dimensions which get higher scores are support (given to the teachers) and organization (coming from the board of directors).

Keywords

accompaniment; boards of directors; satisfaction; skill

\section{Introducción}

Diversas investigaciones muestran que la labor de los directivos es fundamental para lograr mejores resultados en los centros educativos. Los líderes de estas instituciones, con características tanto personales: empatía, comprensión, habilidades comunicacionales, iniciativa, capacidad de enseñar y de interesarse por el otro, madurez emocional y social; como técnicas: conocimientos pedagógicos, dominio de técnicas grupales, de relaciones humanas, conocimientos administrativos, de gestión y de metodologías de las diversas áreas, llevadas a la práctica permitirían generar cambios en las organizaciones educativas (Mogollón, 2006).

El acompañamiento directivo se le brinda a los docentes de aula, y su objetivo es entregar orientaciones técnicas, desarrollar sus destrezas y mantener la sensibilidad a través de las relaciones humanas (Brigg, 2000). La labor de acompañamiento implicaría el desarrollo de estas habilidades, pues busca una relación más estrecha de los directivos con la comunidad, a fin de conocer de manera cercana y sistemática las necesidades y demandas de los docentes, y articularse adecuadamente para contribuir a la mejora del clima del centro educativo (Garay \& Uribe, 2006).

Las formas de supervisión que realizan los directivos hacia los docentes son algunas de las dimensiones que afectan e influyen en su satisfacción laboral y en su forma de desempeño
(Barraza \& Ortega, 2009). La satisfacción laboral es un estado afectivo positivo y placentero, que manifiesta la persona hacia su respectivo ambiente de trabajo (De Frutos, González, Maíllo, Peña, \& Riesco, 2007), y puede tener múltiples dimensiones. Para efectos de esta investigación, se considerará la dimensión de satisfacción laboral específicamente referida a las relaciones entre docentes y directivos, pues uno de los factores que incide negativamente en la satisfacción laboral de los docentes es la forma en la que son supervisados (Yañez, Arenas, \& Ripoll, 2010; Jaik, Tena, \& Villanueva, 2010). Son los equipos directivos y especialmente el Director, quienes tienen la misión de mantener el equilibrio en las relaciones interpersonales que se desarrollan en las organizaciones educativas.

Los directivos deben poseer habilidades no solo técnicas, sino primordialmente habilidades sociales, relacionales, contar con valores y actitudes acordes a lo que implica el trabajo con personas (Ariza \& Ocampo, 2005).Por lo expuesto, existe la necesidad de generar un programa que desarrolle las habilidades de los equipos directivos para acompañar al docente en su quehacer pedagógico. El fundamento de este programa se basa en las teorías de la autonomía de Deci y Ryan (1985), la teoría del aprendizaje (reforzamiento de comportamientos deseables) y el enfoque de la terapia interaccional estratégica (Haley, 1980; Hirsch \& Rosarios, 1987). La teoría de la autonomía sobre la motivación humana analiza el desarrollo y funcionamiento de la personalidad en contextos sociales, el grado en que las conductas humanas son volitivas o autodeterminadas; es decir, el grado en que las personas realizan sus acciones al nivel más alto de reflexión y se comprometen con un sentido de elección (Deci \& Ryan, 1985). Una necesidad psicológica básica es tener relaciones interpersonales significativas con otros para, si estas se satisfacen, generar en las personas motivación intrínseca y satisfacción (Deci \& Ryan, 2008); el aprendizaje, implica un cambio en la conducta, que es perdurable y se obtiene de las experiencias y prácticas (Ertmer \& Newby, 1993). 
La terapia interaccional estratégica está orientada a resolver situaciones y problemas. Esta plantea la existencia de un agente de cambio quien prepara un diseño específico para cada situación o problema, toma la iniciativa que permitirá orientar la terapia e influir directamente sobre el(los) consultante(s) (Haley, 1980). Un procedimiento es estratégico, cuando se plantean metas y una planificación para alcanzarlas (Pérez \& Díaz, 2004). Estos postulados entregan bases para planificar una estrategia de intervención (programa de acompañamiento) y definir procedimientos considerando el contexto en el que se desarrolla una intervención.

La presente investigación plantea un programa de acompañamiento directivo con cuatro dimensiones: (1) apoyo afectivo al profesor; (2) valoración del desempeño educativo del profesor; (3) definición de un foco de colaboración con las necesidades educativas del profesor; (4) acompañamiento continuo y sistemático al profesor. Cada dimensión involucra 5 acciones específicas que los directivos deben realizar con los docentes.

1. Brindar apoyo afectivo al profesor.

Si el docente se siente acompañado afectivamente en el centro educativo, probablemente aumentará su motivación y mejorará el clima social escolar. Para esto el directivo tendrá que ir más allá de su labor administrativa, y generar espacios de conversación y confianza en los docentes de aula, así como tiempos para que expresen sus necesidades tanto personales como profesionales (Majluf \& Hurtado, 2008).

El trabajo educativo involucra exigencias físicas, emocionales y sociales, por lo que las relaciones personales y el contacto afectivo que establecen el directivo y los docentes resultan determinantes en el proceso educativo. Las relaciones afectivas que se dan entre los profesores, sus compañeros y sus alumnos, ya sea al interior o fuera del aula, idealmente deberían constituirse en un aporte para la mejora del clima social escolar (Arón \& Milicic, 1999).

2. Valorar el desempeño educativo del profesor.

El reconocimiento y la valoración son considerados fundamentales para mejorar los ambientes de trabajo, por sobre las críticas y el castigo (Arón \& Milicic, 1999). Al reconocer y valorar la labor educativa tanto instructiva como formativa que el docente realiza, el directivo estará ofreciendo motivación (Deci \& Ryan, 1985), lo que ayudará a mejorar sus prácticas pedagógicas. La valoración y reconocimiento de los directivos hacia los docentes puede darse en diversos contextos educativos, tales como la sala de clases, recreos, reuniones de apoderados, reuniones de docentes, entre otros (Majluf \& Hurtado, 2008).

3. Acordar un foco de colaboración con las necesidades educativas del profesor.

Los docentes enfrentan múltiples problemas y dificultades, tanto en la sala de clases como también fuera de ella. El presente enfoque de intervención se plantea considerando las necesidades particulares de cada docente. Se requiere por tanto una intervención estratégica que incluya un procedimiento, metas que apunten a resolver un problema, y una planificación acordada con el profesor que permita lograrlas (Pérez \& Díaz, 2004).

En el acompañamiento que plantea el grupo de directivos, como agentes de cambio, se les invita a hacerse responsables en conjunto de ayudar al docente a resolver sus necesidades educativas específicas, centrándose en alguna relevante para el profesor. Desde esta perspectiva, la influencia de los directivos a través de la intervención estratégica tiene el objeto de provocar cambios (Pérez \& Díaz, 2004).

4. 4. Acompañamiento continuo y sistemático al profesor.

Para que el acompañamiento pueda ser efectivo debe existir un trabajo 
colaborativo y sistemático que aborde las necesidades de los docentes de una forma organizada, adecuada y conociendo la demanda que el profesor requiere. Esto se ajusta a la sistematicidad y organización que plantea el enfoque de la intervención estratégica en que se definen metas, se diseña un plan de acción, se actúa, evalúa y retroalimenta. Este acompañamiento directivo sistemático y continuo se fundamenta en las siguientes características de la intervención estratégica: es una intervención breve, las metas se plantean a corto plazo (en general no más de 6 meses), la acción se concentra directamente en los problemas antes que en las causas remotas, se enfatiza el proceso y la continuidad del mismo antes que el contenido, se efectúan seguimientos regulares para garantizar que el cambio positivo continúe y se realiza evaluación constante del proceso (Haley, 1980 citado en Pérez \& Díaz, 2004).

De acuerdo a lo anterior, el objetivo de esta investigación es valorar la influencia del programa de acompañamiento en la percepción de los docentes y directivos hacia la labor de acompañamiento que realiza el equipo directivo y la satisfacción laboral. Las hipótesis que se plantean, sobre la aplicación del Programa de acompañamiento directivo (PAD) son:

1. El PAD mejora la satisfacción laboral y la percepción de los docentes hacia la labor de acompañamiento realizada por los directivos del centro educativo.

2. El PAD mejora la percepción de los directivos del centro educativo hacia su labor de acompañamiento al docente y la satisfacción percibida en los docentes.

\section{Método}

\section{Diseño}

Se aplicó un diseño cuasi experimental con mediciones en el grupo experimental (21 sujetos) y en el grupo control (7 sujetos) pre y post intervención.

Aplicación en el grupo experimental: el programa se realizó por un período de cinco semanas, en el cual los directivos aplicaron las acciones del programa de acompañamiento a los docentes, quienes fueron asesorados durante el proceso por una facilitadora.

La escuela que sirvió como modelo de comparación no recibió entrenamiento pero, se aplicaron mediciones pre y post intervención.

\section{Participantes}

Los participantes son 12 directivos y 16 docentes de centros educativos con un alto índice de vulnerabilidad socioeconómica, de enseñanza básica y media, de la Región del Bío-Bío, Chile.

Se seleccionaron cuatro centros educativos de forma intencionada, se consideró accesibilidad, que fuesen colegios urbanos. Se asignaron tres centros a la condición de experimental y uno a la condición de comparación (control).

En cada centro educativo participaron los tres directivos (Director, Jefe de Unidad Técnico Pedagógica e Inspector General) y cuatro docentes de quinto año de educación básica.

La totalidad de los directivos participantes contaban con especialización en administración educacional y dos de ellos poseían grado de magíster. Eran 11 hombres y 1 mujer, las edades fluctuaron entre los 30 y 50 años de edad, y contaban con más de 5 años de experiencia de trabajo en gestión educacional. Los docentes participantes fueron 7 mujeres y 6 hombres, con especialización en sus respectivas asignaturas, sin estudios de postgrado, las edades fluctuaron entre los 30 y 50 años de edad, y contaban con más de 5 años de experiencia de trabajo en su área. 


\section{Instrumentos de medida}

\section{Cuestionario de Percepción del Acompañamiento Directivo (CPLAD)}

Para medir la percepción del acompañamiento, se utilizó el Cuestionario de percepción de la labor de acompañamiento directivo (CPLAD), el cual posee dos formas de aplicación, una para directivos y otra para docentes. Contiene 16 ítems distribuidos en cuatro dimensiones: apoyo afectivo, valoración, foco de colaboración y sistematicidad. Para cada dimensión hay cuatro ítems. Tanto para la forma de directivos como de docentes las preguntas 1, 5, 9 y 13 corresponden a la dimensión apoyo afectivo, las preguntas 2, 6,10 y 14 corresponden a la valoración del trabajo del profesor, las preguntas 3, 4, 7 y 15 corresponden a foco de colaboración y las preguntas $8,11,12$ y 16 corresponden a sistematicidad. Cada ítem corresponde a una pregunta sobre la frecuencia con que el directivo realiza una acción de acompañamiento (que corresponde a las acciones del programa de acompañamiento que el directivo realiza con los docentes). Cada ítem tiene cuatro alternativas de respuesta: "Nunca”, "1 vez por semana", "2 veces por semana", "3 o más veces por semana".

La consistencia interna del CPLAD - directivos y del CPLAD-docentes se muestra en la tabla 1.

\section{TABLA 1}

Alfa de Cronbach CPLAD-Directivos y CPLADDocentes

\begin{tabular}{lllll}
\hline Dimensión & Director & $\begin{array}{c}\text { Docentes } \\
\text { Jefe de UTP }\end{array}$ & Inspector general & Equipo directivo \\
\hline Total & 0.94 & 0.96 & 0.95 & 0.77 \\
Apoyo afectivo & 0.88 & 0.89 & 0.9 & 0.78 \\
Valoración & 0.82 & 0.9 & 0.78 & 0.79 \\
Foco & 0.77 & 0.81 & 0.77 & 0.65 \\
Sistematicidad & 0.7 & 0.79 & 0.62 \\
\hline \multicolumn{5}{c}{ Fuente: } \\
\hline \multicolumn{5}{c}{ elaboración propia. }
\end{tabular}

Se realizó la validación de contenidos del CPLAD mediante opinión de cinco jueces expertos, quienes contaban con 5 años de experiencia en cargos directivos, al menos un curso de postítulo o de especialización en el área de gestión y al menos cinco años de experiencia en el aula.
Dimensión Equipo Directivo de la Escala Multidimensional de Satisfacción Laboral Docente de Barraza y Ortega (EMSLD) (adaptada para el presente proyecto de investigación)

La escala original (Barraza \& Ortega, 2009) contiene 34 ítems distribuidos en ocho dimensiones. Para medir la satisfacción laboral de los docentes sobre el acompañamiento que los directivos realizan a los docentes, se utilizarán los ítems de Relaciones interpersonales y Equipo directivo. Se utilizarán cinco ítems. Se adaptó una forma para utilizar los directivos con los mismos ítems. La escala obtuvo un Alfa de Cronbach 0.83. Cada ítem corresponde a una pregunta sobre el grado de satisfacción laboral de los docentes, relativa al acompañamiento directivo, el cual tiene cuatro alternativas de respuesta que son: "Totalmente Insatisfecho" (0 puntos), "Algo Insatisfecho" (1 punto), "Algo Satisfecho" (2 puntos) y "Totalmente Satisfecho" (3 puntos). El puntaje final corresponde a la suma de los puntajes de todos los ítems.

\section{Procedimientos}

\section{Procedimientos y aplicación del Programa}

El equipo directivo (Director, Inspector General, Jefe de UTP) de la comuna de Quilleco, Región del Bío-Bío, debía desarrollar con sus docentes de quinto año básico, las acciones que el programa contenía para cada uno de los cuatro ámbitos, y llevar un registro de ello. El proceso de acompañamiento era seguido y guiado por una facilitadora que se reunía con el equipo directivo en dos tipos de sesiones: (1) con los directivos en cada escuela y (2) con los equipos directivos de todas las escuelas intervenidas.

La aplicación del programa duró cinco semanas distribuidas de la siguiente forma: 
Reuniones con facilitadora, quien siguió la aplicación del programa:

a) Aplicación de la medición inicial.

b) Una sesión semanal de 1 hora con los 9 directivos (alternadamente en cada establecimiento educativo).

c) Una sesión semanal de 1 hora con los 3 directivos de cada escuela.

Aplicación: realizada por los propios directivos

Cinco horas semanales de trabajo de aplicación del programa: una hora de trabajo diaria (considerando el acompañamiento a los cuatro profesores).

\section{Diagnóstico y evaluación: 2 horas}

Aplicación de la medición post.

En una primera sesión con los directivos, se dio a conocer y explicó la forma de aplicación del programa, en qué consistía el acompañamiento y las formas de registrarlo. Se trabajó en sesiones grupales denominadas generales (con los tres equipos directivos) y específicas (con los directivos de cada escuela). Luego, en las escuelas, cada integrante del equipo directivo planificada y coordinadamente con sus otros 2 colegas directivos desarrolló las acciones del Programa de acompañamiento directivo (PAD).

\section{Procedimientos de análisis de datos}

Se estudiaron las características psicométricas básicas de los instrumentos, específicamente la consistencia interna medida con el Alfa de Cronbach. Se describió el resultado por dimensiones del Cuestionario de percepción de acompañamiento directivo (CPLAD) tanto para los docentes como para los directivos y del Cuestionario de satisfacción, utilizando la media aritmética y la desviación estándar para los resultados del pre y post-test y de las diferencias entre ambos. Se analizaron los resultados del pre y post test para la evaluación del efecto de la aplicación del PAD usando análisis no paramétrico basado en estadísticos de orden. Posteriormente, se utilizó un análisis no paramétrico, por el número reducido de datos que impidió el uso de pruebas paramétricas basadas en el teorema de límite central, y porque en la exploración de los datos se observó que las distribuciones por escala no eran normales. Específicamente, se utilizó un análisis no paramétrico basado en estadísticos de orden para datos longitudinales en diseños factoriales usando la librería n-parLD (Noguchi, Gel, Brunner, \& Konietschke, 2012).

Se consideró un diseño de medidas repetidas sobre un factor correspondiente a la escuela. Se utilizó la prueba ATS, equivalente en un análisis paramétrico a un ANOVA de dos vías y con bloques, que correspondía a las vías al tiempo, la escuela, los bloques y los sujetos. La prueba ATS, en un primer momento comprueba la existencia de un efecto significativo de interacción entre la escuela y el tiempo, el cual indicaría que por lo menos hubo un cambio en uno de los cuatro centros educativos entre el pre y posttest. En un segundo momento se verifica que la escuela que tenga menores puntajes en el posttest sea la escuela que no se intervino, para esto específicamente se utilizaron los efectos relativos, entendidos como la probabilidad de que un sujeto correspondiente a un determinado grupo posea un valor igual o superior al de cualquier otro sujeto elegido al azar de la muestra (Noguchi et al., 2012). Finalmente se comprueba la existencia de diferencias significativas en el posttest, entre la escuela que no se intervino (grupo comparación) en contraste con los tres centros educativos en que sí se aplicó el programa. Para esto se utiliza una prueba de contraste de efectos relativos que contrasta la escuela 1 o grupo comparación con cada una de las tres escuelas intervenidas.

Debido al alto número de pruebas estadísticas utilizadas, para controlar el error de tipo I, se presenta el resultado del método de Holm para ajuste del error tipo I por familias de análisis. 


\section{Resultados}

\section{Valoración del Programa de Acompañamiento}

Se afirma la primera hipótesis: "la aplicación de un Programa de acompañamiento directivo mejora la satisfacción laboral y la percepción de los docentes hacia la labor de acompañamiento realizada por los directivos del centro educativo" porque mejoró la satisfacción laboral y la percepción de los docentes sobre la labor de acompañamiento en los tres centros educativos en los que se aplicó el PAD. Esto se verifica con los efectos relativos, (probabilidad de que un sujeto correspondiente a un determinado grupo posea un valor igual o superior al de cualquier otro sujeto elegido al azar de la muestra).

En la tabla 2 se presentan los efectos relativos para las distintas dimensiones del PAD por directivo, obtenidos del CPLAD. docentes. No existen diferencias significativas en las cuatro dimensiones del programa para el Director. Para el Inspector General se observan diferencias significativas solo en las dimensiones de valoración y foco. En valoración, se presentan diferencias en las escuelas 3 y 4 , por sobre el grupo comparación y en foco, en todas las escuelas por sobre el grupo comparación. Para el Jefe de UTP se presentan diferencias significativas en las cuatro dimensiones y en las tres escuelas en que se aplicó el PAD.

Para la variable satisfacción laboral existen diferencias significativas en las tres escuelas intervenidas.
TABLA 2

Efectos Relativos post-test y valor-p de la prueba ATS del PAD ajustados, en la percepción sobre la labor de acompañamiento y la satisfacción de los docentes

\begin{tabular}{cccccc}
\hline $\begin{array}{c}\text { Dimensión por } \\
\text { directo }\end{array}$ & Escuela 1 & Escuela 2 & Escuela 3 & Escuela 4 & $\begin{array}{c}\text { Valor p, } \\
\text { prueba ATS } \\
\text { ajustada }\end{array}$ \\
\hline $\begin{array}{c}\text { Acompañamiento } \\
\text { Director }\end{array}$ & & & & & \\
$\begin{array}{c}\text { A poyo afectivo } \\
\text { Valoración }\end{array}$ & 0.35 & 0.79 & 0.85 & 0.79 & 0.08 \\
$\quad 0.55$ & 0.75 & 0.82 & 0.86 & 0.06 \\
$\quad$ Foco & 0.37 & 0.77 & 0.77 & 0.85 & 0.06 \\
Sistematicidad & 0.54 & 0.75 & 0.8 & 0.88 & 0.08 \\
Inspector general & & & & & \\
Apoyo afectivo & 0.35 & 0.82 & 0.83 & 0.79 & 0.06 \\
Valoración & 0.48 & 0.74 & $0.85^{*}$ & $0.85^{*}$ & 0.02 \\
$\quad$ Foco & 0.39 & $0.76^{*}$ & $0.82^{*}$ & $0.86^{*}$ & 0 \\
Sistematicidad & 0.46 & 0.74 & 0.8 & 0.87 & 0.06 \\
Jefe de U TP & & & & & \\
A poyo afectivo & 0.34 & $0.81^{*}$ & $0.78^{*}$ & $0.85^{*}$ & 0 \\
Valoración & 0.36 & $0.79^{*}$ & $0.77^{*}$ & $0.88^{*}$ & 0 \\
Foco & 0.38 & $0.78^{*}$ & $0.8^{*}$ & $0.86^{*}$ & 0 \\
Sistematicidad & 0.41 & $0.71^{*}$ & $0.82^{*}$ & $0.88^{*}$ & 0.01 \\
\hline Satisfacción & 0.56 & $0.75^{*}$ & $0.76^{*}$ & $0.86^{*}$ & 0 \\
\hline & & & & & \\
\hline
\end{tabular}

Notas: * Diferencia significativa de efectos relativos de las escuelas con respecto a la escuela 1 (grupo comparación). La prueba ATS muestra la presencia de diferencia entre los colegios en el post-test, con valores menores a 0.05 Fuente: elaboración propia.

En la hipótesis 2, se afirma que: "La aplicación de un programa de acompañamiento directivo mejora la percepción de los directivos del centro educativo hacia su labor de acompañamiento al docente y la satisfacción percibida en los docentes". Lo que se puede corroborar luego del siguiente análisis del CPLAD y escala de satisfacción directivos.

En la tabla 3 se observan diferencias significativas en los valores de $\mathrm{p}$ en dos de las cuatro dimensiones que corresponden a valoración en las escuelas 3 y 4 y sistematicidad en todas las escuelas por sobre el grupo comparación. Para la variable satisfacción existe diferencia significativa y esta se produce en las escuelas 2 y 4 por sobre el grupo comparación. 
VALORACIÓN DE LA INFLUENCIA DE UN PROGRAMA DE ACOMPAÑAMIENTO DIRECTIVO SOBRE LA PERCEPCIÓN Y...

sistematicidad. El desarrollo del PAD mejora la satisfacción percibida por los directivos en los docentes y las mejores puntuaciones se presentan en las escuelas 2 y 4.

El modelo del Sistema de Aseguramiento de la Calidad de la Educación (SACGE), considera al Director y al equipo directivo como actores claves para lograr buenos resultados organizacionales en las instituciones educativas, tanto en aspectos de climas organizacionales como en la enseñanza en sí. Se plantea un equilibrio entre el trabajo administrativo y de gestión de relaciones, manteniendo un clima de confianza y armonía (Ahumada, Galdames, González, \& Herrera, 2009). En este programa se busca lograr este equilibrio y los resultados muestran una actitud crítica de los propios directivos hacia su labor de acompañamiento al docente. Una clave para modificar procesos de instrucción en el aula que contribuya a la mejora de los aprendizajes, es participar en los procesos que se desarrollan al interior de esta, realizando visitas en forma sistemática no solo para realizar una supervisión formal sino para apoyarlos y conversar con los docentes y los estudiantes. Estas visitas se complementan con las reflexiones entre docentes y directivos acerca de sus acciones en el aula y de los aprendizajes de los alumnos (Anderson, 2010). En este contexto, el PAD que se ha planteado, no solo contribuye a mejorar las relaciones interpersonales, reflejadas en la mejora de la satisfacción laboral, sino que busca mejorar los aprendizajes de los estudiantes por medio del desarrollo de habilidades directivas que involucren el acompañamiento a los docentes.

Una limitación del estudio es la representatividad de la muestra, lo que se podría mejorar en futuras investigaciones. Las proyecciones de esta investigación se orientan a mejorar el desarrollo del Programa de acompañamiento con el director, quien obtuvo las menores puntuaciones pos-test. Esto considera el acercamiento de los directivos a los docentes para mejorar las relaciones interpersonales y sistematizar el acompañamiento de los directivos hacia lo docentes, existiendo mejora en las relaciones resulta más fácil generar instancias efectivas de acompañamiento y sistematizarlas en el tiempo.

\section{Agradecimientos}

Financiamiento de Conicyt.

\section{Referencias}

Ahumada, L., Galdames, S., González, A., \& Herrera, P. (2009). El funcionamiento del Equipo Directivo durante un proceso de autoevaluación institucional en el marco de políticas de Aseguramiento de la Calidad de la Gestión Escolar en Chile. Universitas Psychologica 8(2), 353-370.

Anderson, S. (2010). Liderazgo directivo: Claves para una mejor escuela. Psicoperspectivas, 9(2), 34-52.

Ariza, G. \& Ocampo, H. (2005). El acompañamiento tutorial como estrategia de a formación personal y profesional. Universitas Psychologica, 4(1), 31-41.

Arón, A. M. \& Milicic., N. (1999). Clima social escolar y desarrollo personal. Santiago: Andrés Bello.

Barraza, A. \& Ortega, F. (2009). Satisfacción laboral en instituciones formadoras de docentes. Un primer acercamiento. Revista Diálogos Educativos, 9(17), 4-174. Extraído Recuperado el 20 de Junio del 2011 de http://www.umce.cl/ dialogos/n17_200 9/barraza.swf

Brigg, L. (2000). La supervisión. México: Ed. McGraw-Hill.

Deci, E. \& Ryan, R. (1985). The general causality orientations scale: Self-determination in personality. Journal of Research in Personality, 19(2), 109-134.

Deci, E. \& Ryan, R. (2008). Self-determination theory: A macrotheory of human motivation, development, and health. Canadian Psychology, 49(3), 182-185.

De Frutos, J., González, P., Maíllo, A., Peña, J., \& Riesco, M. (2007). Condiciones de trabajo y satisfacción laboral de los docentes en 
las Escuelas Católicas de Madrid. Revista Educación y Futuro, 17, 9-42.

Garay, S. \& Uribe, M. (2006). Dirección escolar como factor de eficacia y cambio en educación: situación de la dirección escolar en Chile. Revista Electrónica Iberoamericana sobre Calidad, Eficacia y Cambio en Educación,4(4e), 39-64.

Haley, J. (1980). Terapia para resolver problemas. Buenos Aires: Amorrortu.

Hirsch, H. \& Rosarios, H. (1987). Estrategias psicoterapéuticas institucionales. La organización del cambio. Buenos Aires: Nadir.

Jaik, A., Tena, J. \& Villanueva, R. (2010). Satisfacción laboral y compromiso institucional de los docentes de posgrado. Revista Diálogos Educativos, 19, 119-130.

Majluf, N. \& Hurtado, J. (2008). Hacia una mejor gestión en los colegios. Influencia de la cultura escolar sobre la educación. Santiago: Pontificia Universidad Católica.

Miranda, E. (2002). La supervisión escolar y el cambio educativo. Un modelo de supervisión para la transformación, desarrollo y mejora de los centros. Revista de currículum y formación del profesorado, 6(1-2), 1-13.

Mogollón, A. (2006). Calidad y enfoques de la supervisión. Revista Ciencias de la Educación, 1(23), 29-46.

Noguchi, K., Gel, Y.R., Brunner, E., \& Konietschke, F. (2012). nparLD: An R Software Package for the Nonparametric Analysis of Longitudinal Data in Factorial Experiments. Journal of Statistical Software, 50(12), 1-23. http://dx.doi.org/10.18637/jss .$v 050.112$

Ertmer, P., \& Newby, T. J. (1993). Conductismo, cognitivismo y constructivismo: una comparación de los aspectos críticos desde la perspectiva del diseño de instrucción. Performance Improvement Quarterly, 6(4), 50-72

Pérez, M.V. \& Díaz, A. (2004). Recursos para motivar al estudio, Reflexiones para la Consejería Educacional y Vocacional. Concepción: Universidad de Concepción,
Facultad de Educación, Dirección de Educación Continua.

Yañez G. R., Arenas C. M., \& Ripoll N. M. (2010). El impacto de las relaciones interpersonales en la Satisfacción laboral general. Liberabit, 16(2),193-201.

\section{Notas}

* Artículo de investigación. 